\title{
EGMlab, a scientific software for determining the gravity and gradient components from global geopotential models
}

\author{
R. Kiamehr • M. Eshagh
}

Received: 8 December 2007 / Accepted: 7 July 2008 / Published online: 12 August 2008

(C) Springer-Verlag 2008

\begin{abstract}
Nowadays, Global Geopotential Models (GGMs) are used as a routine stage in the procedures to compute a gravimetric geoid. The GGMs based geoidal height also can be used for GPS/levelling and navigation purposes in developing countries which do not have accurate gravimetric geoid models. Also, the GGM based gravity anomaly including the digital elevation model can be used in evaluation and outlier detections schemes of the ground gravity anomaly data. Further, the deflection of vertical and gravity gradients components from the GGMs can be used for different geodetic and geophysical interpretation purposes. However, still a complete and user-friendly software package is not available for universities and geosciences communities. In this article, first we review the procedure for determination of the basic gravity field and gradient components from the GGMs, then general MATLAB based software is presented and applied as a sample case study for determination of gravity components based on the most recent EIGEN-GL04C GRACE model in Sweden.
\end{abstract}

Communicated by: H. A. Babaie

Electronic supplementary material The online version of this article (doi:10.1007/s12145-008-0013-4) contains supplementary material, which is available to authorized users.

R. Kiamehr $(\bowtie)$

Department of Geodesy and Geomatics, Zanjan University,

45195-313 Zanjan, Iran

e-mail: ramin@znu.ac.ir

\section{Eshagh}

Division of Geodesy, Royal Institute of Technology,

SE10044 Stockholm, Sweden

e-mail: eshagh@kth.se
Keywords Deflection of vertical components · GPS · Geoid · Geoidal height · Global geopotential model · Gravity anomaly $\cdot$ Gravity gradients $\cdot$ Levelling $\cdot$ Sweden

\section{Introduction}

In the 1960s and 1970s, satellite geodesy provided geodesists with global geopotential models (GGMs), which are spherical harmonic representations of the long and medium wavelength $(>100 \mathrm{~km})$ components of the earth's gravity field. The new satellite gravity missions CHAMP and GRACE lead to significant improvements of our knowledge about the long wavelength part of the Earth's gravity field, and thereby of the long wavelengths of the geoid. They provide a homogeneous and near-complete global coverage of gravity field information. However, frequently the error estimates for GGMs are too optimistic and/or presented as global averages and thus not necessarily representative of the performance of the GGM in a particular region. Hence, the user of a GGM should perform his own accuracy and precision verifications, such as comparing the GGM-derived gravity field quantities with local data. So, users of any GGM should perform their own accuracy and precision verifications, such as comparing the GGM-derived gravity field quantities with local data (cf. Kirby et al. 1998).

We know that there are many un-surveyed areas of the Earth. On the oceans we have today gravity anomalies determined from satellite altimetry, but the effects of the ocean circulation and different biases deteriorate this data since today we only have estimates of the (quasi-stationary) sea-surface topography at very long wavelengths. In addition, where we have gravity data, we do not always know the reference system used and sometimes even 
Bouguer-anomalies and free-air anomalies are mixed up. On the contrary, satellite-only solutions are not polluted by surface data like the existing high resolution spherical harmonic models based on data combination.

A variety of global geopotential models, which express the Earth's gravity field and thus geoid heights in terms of spherical harmonic basis functions have been computed by various groups for example GPM98A,B,C (Wenzel 1998), EGM96 (Lemoine et al. 1996) and recent GGMs of CHAMP and GRACE (Prange et al. 2007; Förste et al. 2007). Current models of the Earth's gravitational field can be divided among three primary classes:

Satellite-only GGMs, derived from the tracking of artificial Earth satellites. The well-known models in this category are the GRACE satellite-only models GGM02S (Tapley et al. 2005) and EIGEN-GL04S1 (Förste et al. 2006).

Combined GGMs, derived from a combination of a satellite-only model, terrestrial gravimetry, satellite altimetryderived gravity data in marine areas, and airborne gravimetry. Some well-known GGMs in this group are EGM96, GGM02C (Tapley et al. 2005), EIGEN-GL04C (Förste et al. 2006) and EGM2008 (Pavlis et al. 2008).

Tailored GGMs, derived from a refinement of existing (satellite or combined) GGMs using higher resolution gravity data that may have not necessarily been used previously. In this group, we can mention the GPM89C model of Wenzel (1998).

Having briefly reviewed the mathematical relations of the different gravity field and gravity gradients components, the article gives a general description of the MATLAB software and its applications in generating the parameters in territory of Sweden. Determination of the gravity gradient tensor components from GGMs is a new regional aspect is also considered in this article.

Applications of the GGMs in geodesy and geophysics

The GGM based geoidal heights are used for different purposes in geodetic communities. Precise regional geoid determinations are usually carried out using a global geopotential model, together with a set of point or mean terrestrial gravity anomalies and topographic information. In order to determine the most suitable geopotential model for this combination, it is necessary to check the statistical fit of several high degree models to the gravity field. This comprises the fit of the geopotential models to geometrically derived geoid heights at the study region. The best GGM can be used through combination of the gravity data with the Stokes's formula (Kiamehr 2006; Kiamehr and Sjöberg 2005).

Further, nowadays for many applications, conventional levelling using spirit level is being replaced by height determination using the satellite Global Positioning System (GPS). The GPS technique has been used for levelling projects, e.g., to monitor dam subsidence due to water or natural gas removal, earth crustal movements and to control heights across water bodies in connection with bridge construction. The heights directly derived from GPS measurements are geodetic heights referring to the ellipsoid defined by WGS84. Figure 1 shows the basic relationship between geodetic height $h$, orthometric height $H$ and geoidal height $N$. In the first approximation, the heights are related by:

$h \cong H+N$

The primary problem of transforming GPS-derived geodetic heights into orthometric heights is to determine a reliable geoidal height. This geoidal height can be delivered using either a gravimetric method or a GGM model. In developing countries missing a precise gravimetric geoid model, the GGM derived geoidal height plays an important role to this transformation. Also, the geoidal heights and gravity anomalies have applications in geophysics for studying the interior properties of the earth.

The deflection of vertical components is an angle between normal to the reference ellipsoid and plumb line. This deflection usually is used for reduction of geodetic observations, transformation of coordinates between the astronomical (Featherstone 1999) and geodetic coordinates and geophysical interpretations (Kiamehr 2006) and navigation. Since the deflection of vertical can present the interior density variation it can be used in geophysical exploration. The studies on vertical-horizontal components of the gravity gradient and components of the deflection of

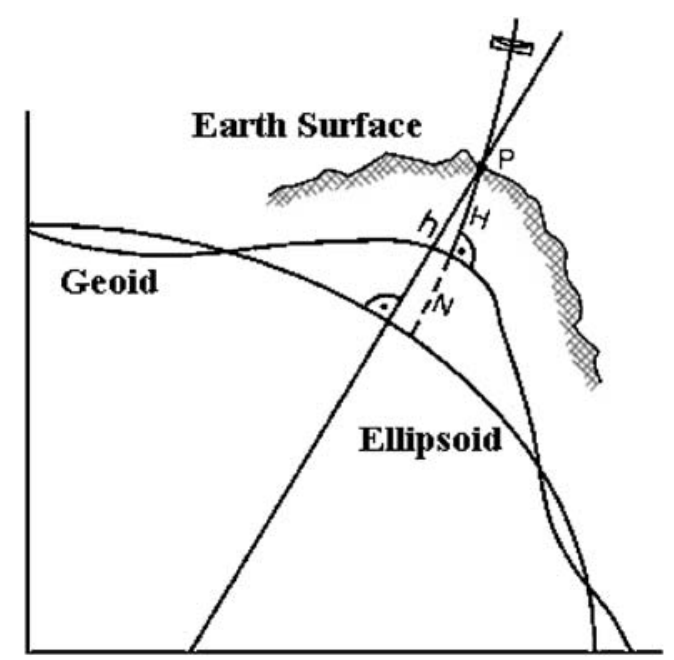

Fig. 1 Principal of levelling with GPS 
vertical by Kiamehr et al. (2008) show very high correlation between these quantities. Also Kiamehr (2006) found a good agreement between tectonic structure and deflection of vertical. Consequently the general pattern of the deflection of vertical computed by a GGM, helps the geoscientists to get some information about lateral density variation.

\section{Gravity field parameters from GGMs}

In the following section we review the basic theories to determine of the gravity and gradient components.

Geoidal height, gravity anomaly and deflection of vertical components

The gravity field has two main structures, long ( $400 \mathrm{~km})$ and short $(25-100 \mathrm{~km})$ wavelengths. The long-wavelengths information can be computed using the satellite orbit perturbation analysis and also terrestrial gravimetric or even satellite altimetry data (Eshagh et al. 2006). The results of such computation are summarized as a Global Geopotential Model (GGM). In geodesy, gravity data are used to define the figure of the Earth. In geophysics, the data are used to constrain subsurface density variations to help to understand problems related to tectonics or commodity exploration. To this end, the geodesist seeks to exploit the differences between the difficult-to-measure real gravity field and a mathematically based model gravity field. Therefore, a mean gravity field should be defined for comparing with the real one. Such gravity field is the normal gravity field, and the residual gravity field is called potential anomaly. The potential anomaly can be used to compute very important quantities relating the physical figure of the Earth to the Mathematical one (ellipsoid) or normal gravity field. Quantities like geoidal height, gravity anomaly and deflection of vertical components are the main quantities. Each one of these quantities expressing the deviation of quantities in normal and real fields can geophysically be interpreted.

The other useful quantity is the gravity gradient of the field which is again referred to the residual fields. Usually the gravity gradients components are coordinated in a North-oriented reference frame. The origin of this frame can be considered on the earth surface or even on the satellite. The $z$-axis is pointing towards the radial direction and upward, $x$-axis points towards the north and the $y$-axis is defined such that the system become right handed in other word, westward.

For global gravity field analysis, the potential anomaly (T) for any interesting point can be represented by a spherical harmonic series, cf. (Heiskanen and Moritz 1967):

$$
\begin{aligned}
& T(r, \varphi, \lambda)=\frac{\mathrm{GM}}{a} \sum_{n=2}^{N_{\max }} \sum_{m=0}^{n}\left(\frac{a}{r}\right)^{n+1}\left(\bar{C}_{n m} \cos m \lambda\right. \\
& \left.+\bar{S}_{n m} \sin m \lambda\right) \bar{P}_{n m}(\cos \varphi),
\end{aligned}
$$

where $r, \varphi, \lambda$, are the geocentric distance, latitude and longitude respectively, GM product of the gravitational constant and Earth's mass, $a$ is the semi major axis of reference ellipsoid, $N_{\max }$ is the maximum degree, $n, m$ stand for degree and order, $\bar{C}_{n m}, \bar{S}_{n m}$ are the normalized geopotential coefficients, and $\bar{P}_{n m}(\cos \theta)$ is the normalized associated Legendre function. According to the Bruns formula (Heiskanen and Moritz 1967, p. 107) we have:

$$
\begin{aligned}
N(r, \varphi, \lambda)=\frac{\mathrm{GM}}{a \gamma} \sum_{n=2}^{N_{\max }} \sum_{m=0}^{n}\left(\frac{a}{r}\right)^{n+1}( & \bar{C}_{n m} \cos m \lambda \\
& \left.+\bar{S}_{n m} \sin m \lambda\right) \bar{P}_{n m}(\sin \varphi),
\end{aligned}
$$

where, $\gamma$ stands for the normal gravity at a certain latitude obtained by Somigliana formula. Also, according to the (Heiskanen and Moritz 1967), one can write the gravity anomaly as a function of geopotential coefficients:

$$
\begin{aligned}
\Delta_{g}(r, \varphi, \lambda)=\frac{\mathrm{GM}}{a} \sum_{n=2}^{N_{\max }} \sum_{m=0}^{n}\left(\frac{a}{r}\right)^{n+1}\left(\frac{n-1}{r}\right)\left(\bar{C}_{n m} \cos m \lambda\right. \\
\left.+\bar{S}_{n m} \sin m \lambda\right) \bar{P}_{n m}(\sin \varphi) .
\end{aligned}
$$

The deflection of the vertical is the angular difference between the direction of the gravity vector $(g)$, or plumb line at a point, and the corresponding ellipsoidal normal through the same point for a particular ellipsoid. The deflection of the vertical has two components (north-south $\xi$ and east-west $\eta$ ). Further, according to Heiskanen and Moritz (1967) the components of deflection of the verticals can be presented as:

$$
\begin{aligned}
\eta(r, \varphi, \lambda)=\frac{\mathrm{GM}}{\operatorname{arr} \sin \varphi} \sum_{n=2}^{N_{\max }} \sum_{m=0}^{n}\left(\frac{a}{r}\right)^{n+1} m\left(\bar{C}_{n m} \sin m \lambda\right. \\
\left.\quad-\bar{S}_{n m} \cos m \lambda\right) \bar{P}_{n m}(\sin \varphi),
\end{aligned}
$$




$$
\begin{aligned}
& \xi(r, \varphi, \lambda)=\frac{\mathrm{GM}}{a r \gamma^{2}} \sum_{n=2}^{N_{\max }} \sum_{m=0}^{n}\left(\frac{a}{r}\right)^{n+1}\left(\bar{C}_{n m} \cos m \lambda+\bar{S}_{n m} \sin m \lambda\right) \times \\
& {\left[\gamma \sqrt{(n-m)(n+m-1)\left(1+\delta_{m}\right)} \bar{P}_{n, m+1}(\sin \varphi)\right.} \\
& \left.-\left(\gamma m \tan \varphi-\frac{d \gamma}{d \varphi}\right) \bar{P}_{n m}(\sin \varphi)\right]
\end{aligned}
$$

where

$$
\frac{\mathrm{d} \gamma}{\mathrm{d} \varphi}=\frac{\gamma_{0} \cos \varphi \sin \varphi}{\left(1-e^{2} \sin ^{2} \varphi\right)^{\frac{3}{2}}}\left(2 k+e^{2}\left(1-k \sin ^{2} \varphi\right)\right),
$$

and $\gamma_{0}$ is the normal gravity at equator, $e^{2}$ is the first eccentricity of the references ellipsoid and $k$ is the constant of formula and $k=0.001931851353$ based on Somigliana's formulas. $\delta$ stands for Kronecker's delta.

Gravity gradients components

The conventional expansion of the gravity gradients in the local North-oriented reference frame have a complicated form, depending on the first and second order derivatives of the associated Legendre functions of the latitude and containing factors which tend to infinity when approaching the poles. In this article, we will use the non-singular expressions for gravity gradients in local north oriented reference frame presented by Petrovskaya and Vershkov (2006). The first and second order derivatives of the disturbing potential $T$ in the north oriented reference frame $\{x, y, z\}$ are considered. We proceed from the known relation (Reed 1973) but we present it in terms of latitude instead of co-latitude:

$$
\begin{aligned}
T_{z z} & =T_{r r}, T_{x x}=\frac{1}{r} T_{r}+\frac{1}{r^{2}} T_{\varphi \varphi}, T_{y y} \\
& =\frac{1}{r} T_{r}+\frac{1}{r^{2} \cot \varphi} T_{\varphi}+\frac{1}{r^{2} \cos ^{2} \varphi} T_{\lambda \lambda} \\
T_{x y} & =\frac{1}{r^{2} \cos \varphi} T_{\varphi \lambda}-\frac{\sin \varphi}{r^{2} \cos ^{2} \varphi} T_{\lambda}, T_{x z} \\
& =\frac{1}{r^{2}} T_{\varphi}-\frac{1}{r} T_{r \varphi}, T_{y z}=\frac{1}{r^{2} \cos \varphi} T_{\lambda}-\frac{1}{r \cos \varphi} T_{r \lambda} .
\end{aligned}
$$

The parameters of gravity gradients have been presented by Petrovskaya and Vershkov (2006) and we present another form of their relations as follow:

$$
\begin{aligned}
T_{z z}=\frac{\mathrm{GM}}{a^{3}} \sum_{n=2}^{N_{\max }} \sum_{m=0}^{n}(n+1)(n+2)\left(\frac{a}{r}\right)^{n+3} & \left(\bar{C}_{n m} \cos m \lambda\right. \\
& \left.+\bar{S}_{n m} \sin m \lambda\right) \bar{P}_{n m}(\sin \varphi)
\end{aligned}
$$

$T_{x x}=\frac{\mathrm{GM}}{a^{3}} \sum_{n=2}^{N_{\max }} \sum_{m=0}^{n}\left(\frac{a}{r}\right)^{n+3}\left(\bar{C}_{n m} \cos m \lambda+\bar{S}_{n m} \sin m \lambda\right) \times$

$$
\begin{gathered}
\left\{a_{n m} \bar{P}_{n m-2}(\sin \varphi)+\left[b_{n m}-(n+1)(n+2)\right] \bar{P}_{n m}(\sin \varphi)\right. \\
\left.+c_{n m} \bar{P}_{n m+2}(\sin \varphi)\right\}, \\
T_{y y}=-\frac{\mathrm{GM}}{a^{3}} \sum_{n=2}^{N_{\max }} \sum_{m=0}^{n}\left(\frac{a}{r}\right)^{n+3}\left(\bar{C}_{n m} \cos m \lambda+\bar{S}_{n m} \sin m \lambda\right) \times \\
\left\{a_{n m} \bar{P}_{n m-2}(\sin \varphi)+b_{n m} \bar{P}_{n m}(\sin \varphi)+c_{n m} \bar{P}_{n m+2}(\sin \varphi)\right\},
\end{gathered}
$$

and

$$
\begin{gathered}
T_{x y}=\frac{\mathrm{GM}}{a^{3}} \sum_{n=2}^{N_{\max }} \sum_{m=1}^{n}\left(\frac{a}{r}\right)^{n+3}\left(\bar{C}_{n m} \sin m \lambda-\bar{S}_{n m} \cos m \lambda\right) \times \\
\left\{d_{n m} \bar{P}_{n-1, m-2}(\sin \varphi)+g_{n m} \bar{P}_{n-1, m}(\sin \varphi)+h_{n m} \bar{P}_{n-1, m+2}(\sin \varphi)\right\} \\
T_{x y}=\frac{\mathrm{GM}}{a^{3}} \sum_{n=2}^{N_{\max }} \sum_{m=0}^{n}\left(\frac{a}{r}\right)^{n+3}\left(\bar{C}_{n m} \cos m \lambda+\bar{S}_{n m} \sin m \lambda\right) \times \\
\left\{\beta_{n m} \bar{P}_{n, m-1}(\sin \varphi)+\gamma_{n m} \bar{P}_{n, m+1}(\sin \varphi)\right\} \\
T_{y z}=\frac{\mathrm{GM}}{a^{3}} \sum_{n=2}^{N_{\max }} \sum_{m=1}^{n}\left(\frac{a}{r}\right)^{n+3}\left(\bar{C}_{n m} \sin m \lambda-\bar{S}_{n m} \cos m \lambda\right) \times \\
\left\{\mu_{n m} \bar{P}_{n-1, m-1}(\sin \varphi)+v_{n m} \bar{P}_{n-1, m+1}(\sin \varphi)\right\}
\end{gathered}
$$

The coefficients of the Legendre functions are numerical constant given in Petrovskaya and Vershkov (2006) p. 126. The gradient components can be used for constructing a geopotential model from the GOCE mission data by the time-wise and space-wise approaches (Petrovskaya and Vershkov 2006). Also, some components (e.g. $T_{z z}$ ) may have a geophysical meaning.

\section{EGMLAB: a MATLAB software for determining the gravity and gradient components from GGMs}

Two MATLAB software programs were developed based on the mentioned formulas in the "Gravity field parameters 
a

Geoid Height, Unit: Meter
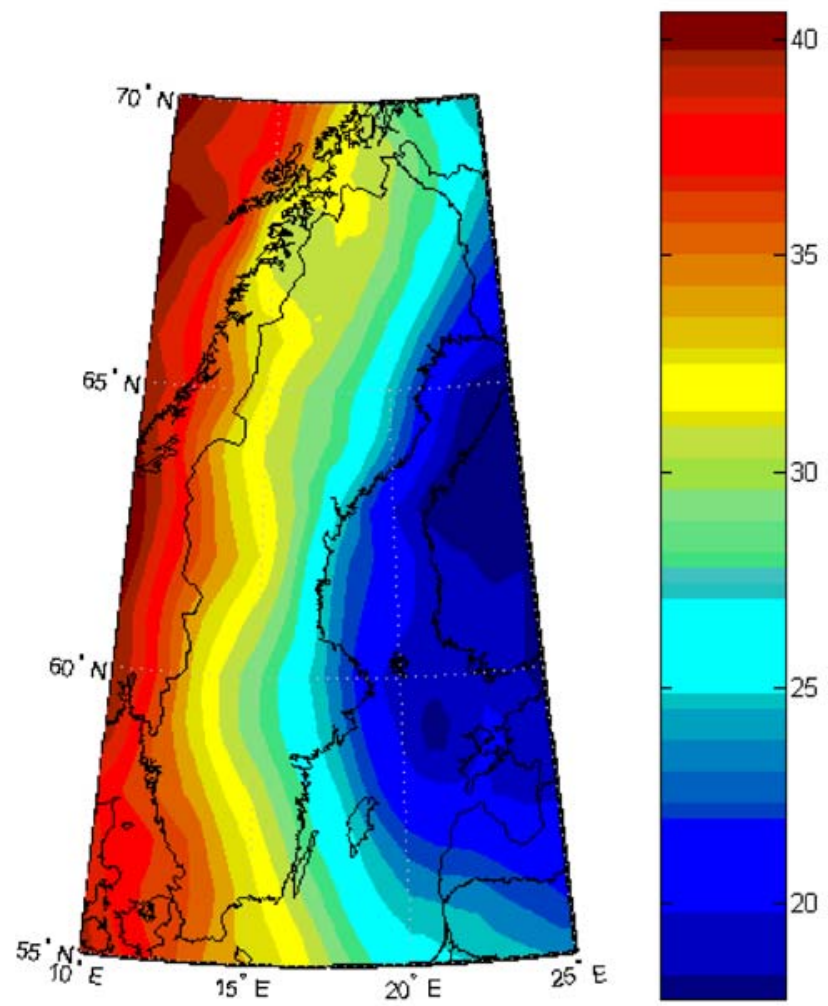

C East-west components of deflection of vertical Unit: arc second
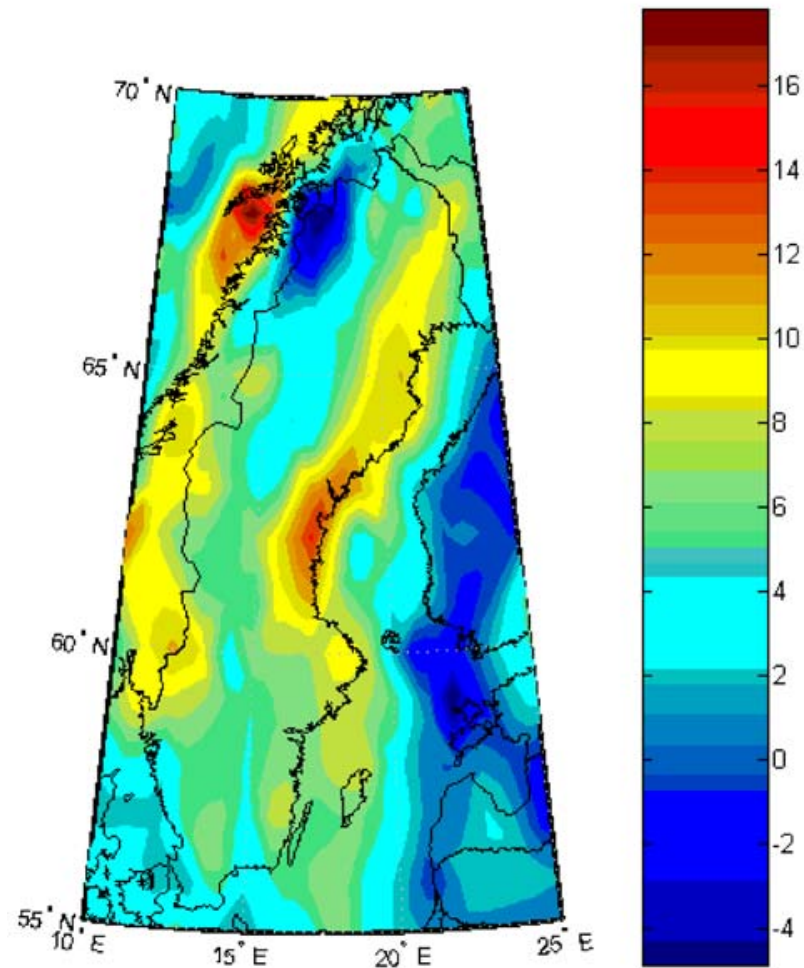

b

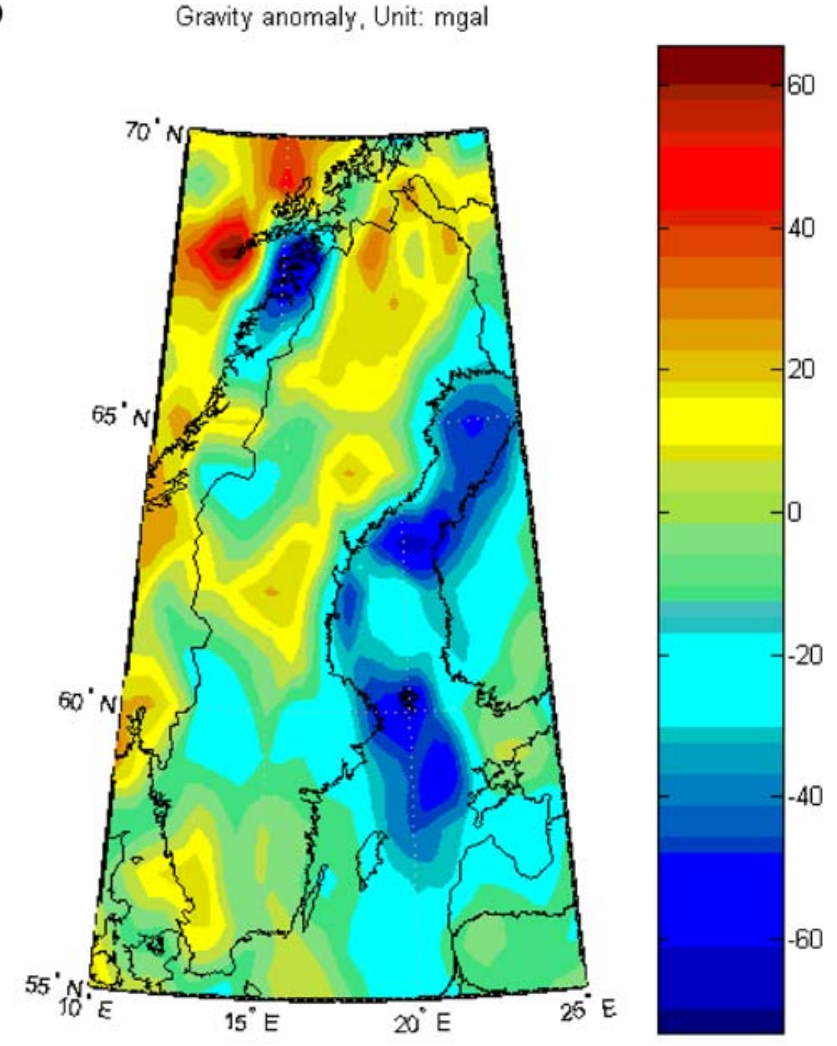

d

North-south component of deflection of vertical Unit: arc second

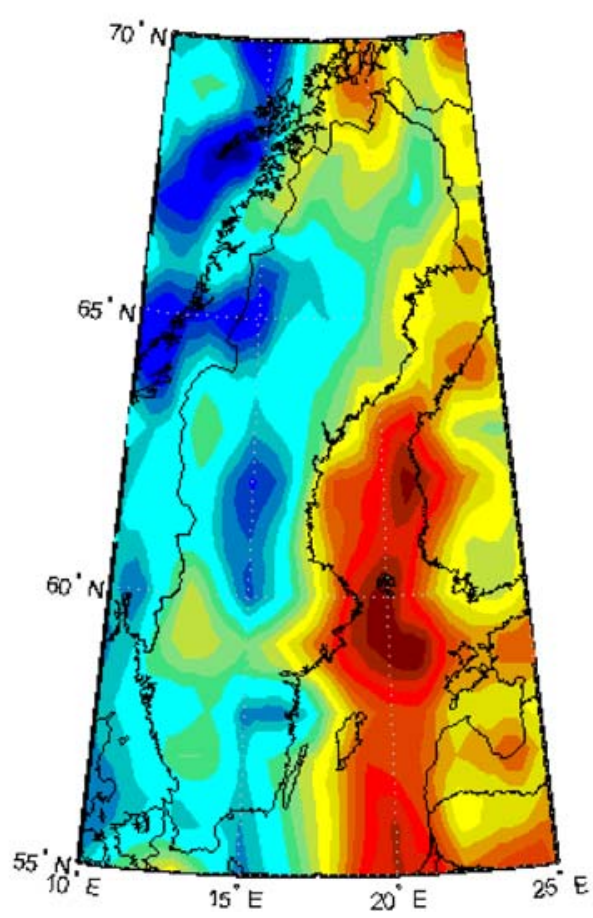

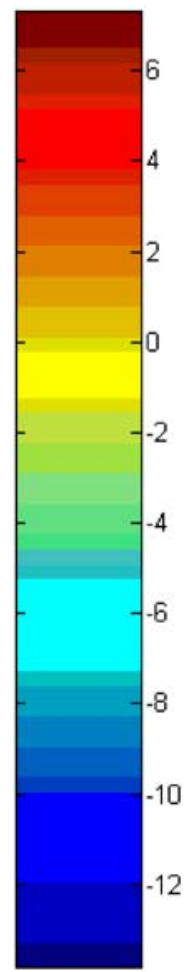

Fig. 2 a Geoidal height, b gravity anomaly and the vertical components $\mathbf{c}$ east-west, $\mathbf{d}$ north-south based on the EIGEN-GL04C model 
Table 1 Statistical analysis of gravity components values in Sweden based on the EIGEN-GL04C model

\begin{tabular}{lrrrr}
\hline Gravity components & Min & Max & Mean & \multicolumn{1}{c}{ Std } \\
\hline$N(\mathrm{~m})$ & 17.585 & 42.017 & 29.288 & 7.113 \\
$\Delta \mathrm{g}(\mathrm{mGal})$ & -81.179 & 78.169 & -3.100 & 24.197 \\
$\xi$ (arc second) & -14.706 & 9.518 & -2.642 & 4.853 \\
$\eta$ (arc second) & -14.706 & 20.820 & -2.642 & 3.866 \\
\hline
\end{tabular}

from GGMs" section. The first program can be used for the computing the anomaly quantities according to Eqs. 3, 4, 5, 6 , and 7 and the second for computation the gravity gradients based on Eqs. 8, 9, and 10. Both of the programs can be used in three different modes, i.e. Point-wise (single point), Block-wise (grid) and Data file. Using the pointwise mode one can compute the anomaly quantities or gravity gradients for one single point; in block-wise mode the user is able to compute the quantities in any region in the world by introducing the coordinates of region borders. The user suffices just to introduce the borders of the block to the program; and in the data file mode the program is performed for some special point which user desires, but the user has to introduce the name of the file containing the latitude and longitude of the expected points. In both programs, the user is asked to choose which mode he/she likes. Depending on the user's choice the program asks the user to introduce the latitude and longitude of the points, in point-wise, block wise and data file containing them.

The user is requested to enter the name of the GGM (e.g. egm96.txt or g104c.txt) in both programs and the output file name for results. The user is able to utilise other GGMs in the software. The spherical harmonics coefficient files for different GGMs can be downloaded from the International Centre for Global Earth Models (ICGEM) website (http:// icgem.gfz-potsdam.de/ICGEM/).

In the case where the block-wise mode is selected, the Isarithmic map of the region will be showed too. The format of the output file comprising the results of computations depends on the program to be used. The programs are started with a help text which clearly explains the necessary input and output parameters and formats.

If the EGMlabl is used the anomaly quantities like geoid height, gravity anomaly and deflection of vertical components (Eqs. 2, 3, 4, 5, and 6) are computed in a file containing six columns, the first two columns stand for latitude and longitude of the points, and the other columns are the geoid height, gravity anomaly, east-west component and north-south components of the vertical deflection in order. The EGMlab3 program is used for computing the gravity gradients components (Eqs. 8, 9, and 10). The output file contains eight columns, the first two columns are the latitude and longitude of the points and the other columns are $T x x, T y y, T z z, T x y, T x z$ and $T y z$, respectively. The EGMlab1 and EGMlab3 are the main program codes, and there are some functions that are used for running such program codes.

\section{- Pnm function}

The Pnm function computed the normalized associated Legendre functions and its first order derivatives using recursion formulae. The input of this function is the latitude and maximum degree and order of polynomials.

\section{- Model read function}

Another function for reading the earth gravity model is also used. This function gets the name of Earth Gravity Model and converts the column wise geopotential coefficients to matrix wise whose rows and columns are related to the degree and order of coefficients. The output of this function is the GM or product of gravitational constant and Earth's mass, Ae is the semi major axis of reference ellipsoid used for the earth gravity model, $N_{\max }$ is the maximum degree of the geopotential model (checked up to degree and order 360) and the matrix-wise geopotential coefficients and their errors.

\section{- Normal function}

Normal function generates the normal gravity field using recursion formulae presented in Heiskanen and Moritz (1967). The normal geopotential coefficients are computed based on GRS80 reference field. However, any geopotential model with different GM can be used in the software as it rescale the geopotential coefficients. It is also important to consider the difference between the GM constant and semi major axis of GRS80 and Earth gravity model. This function includes this consideration. It gets the GM and semi-major axis of the earth gravity model as well as the maximum degree for generation of normal gravity field. The output of this function is the normal geopotential harmonic coefficients.

\section{- Coefficients function}

This function generates the coefficients of the Legendre functions being used for computation of the gravity gradients in Eqs. 8, 9, and 10. This function needs the 
a

Txx,Unit: Eotvos
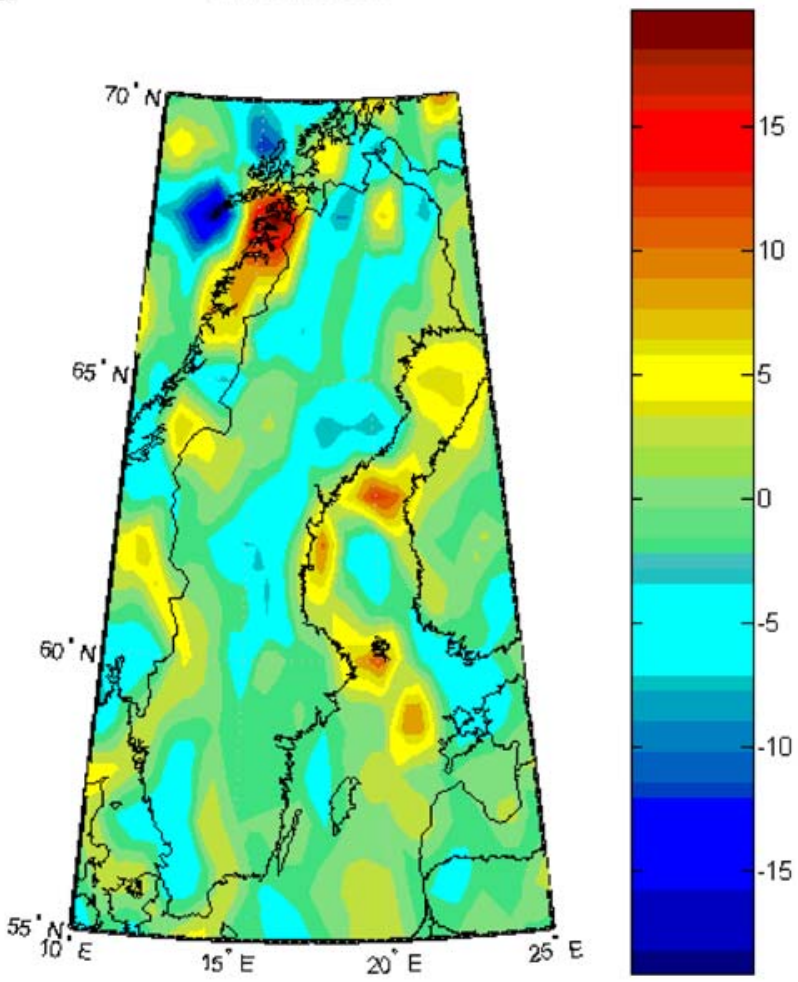

C

Tzz,Unit: Eotvos
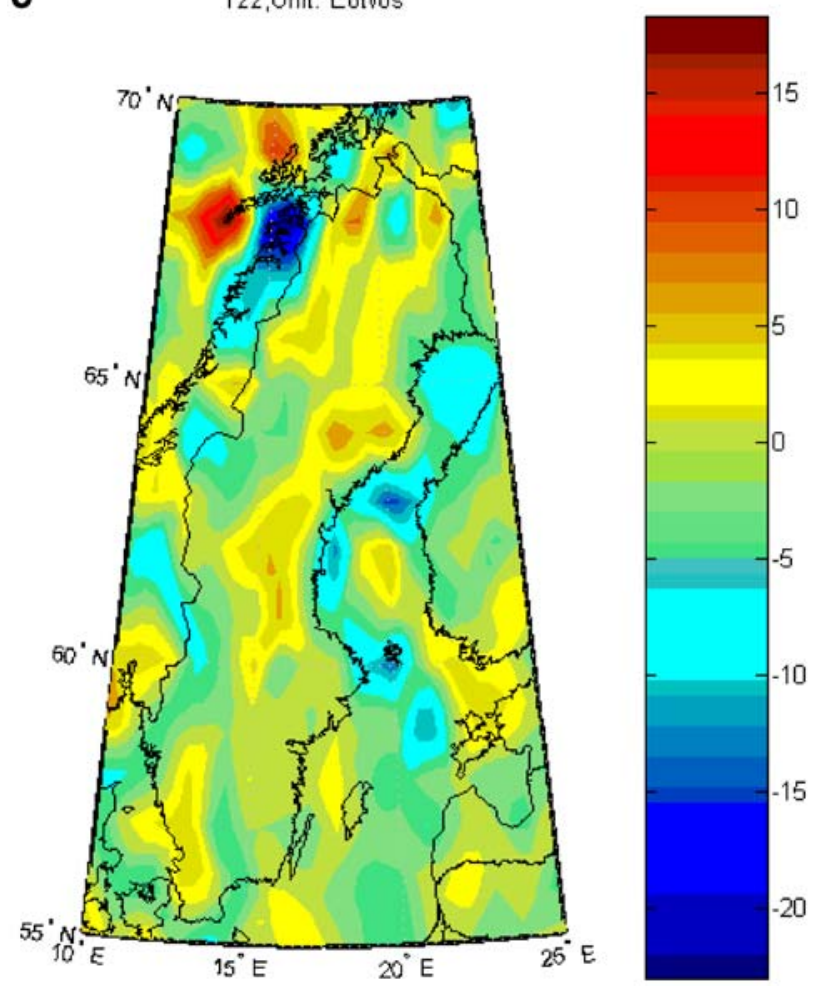

b

Tyy, Unit: Eotvos
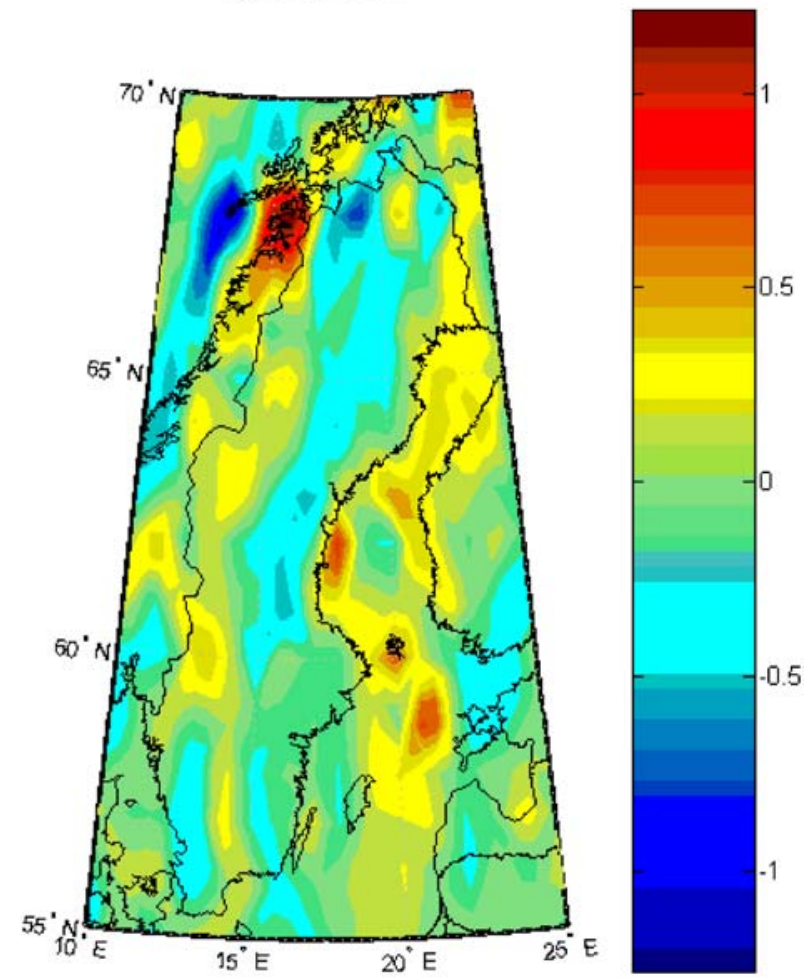

Fig. 3 a Principal gradient components values in Sweden based on the EIGEN-GL04C model. $A T_{x x}, B T_{y y}$, $C T_{z z}$. Unit: Eotvos. b Cross gradient components values in Sweden based on the EIGEN-GL04C model. $D T_{x y}, E T_{x z}, C T_{y z}$. Unit: Eotvos 
d

Txy,Unit: Eotvos

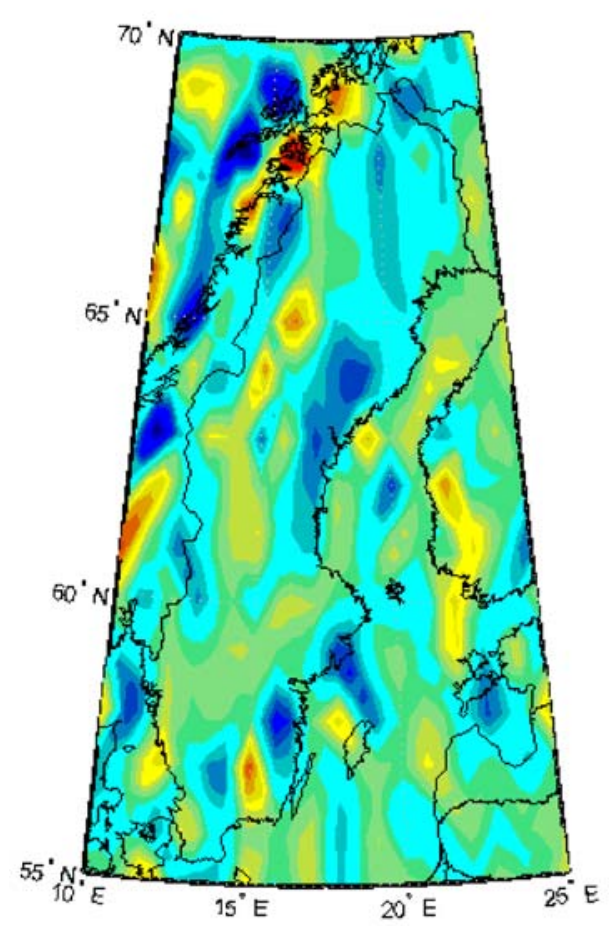

f

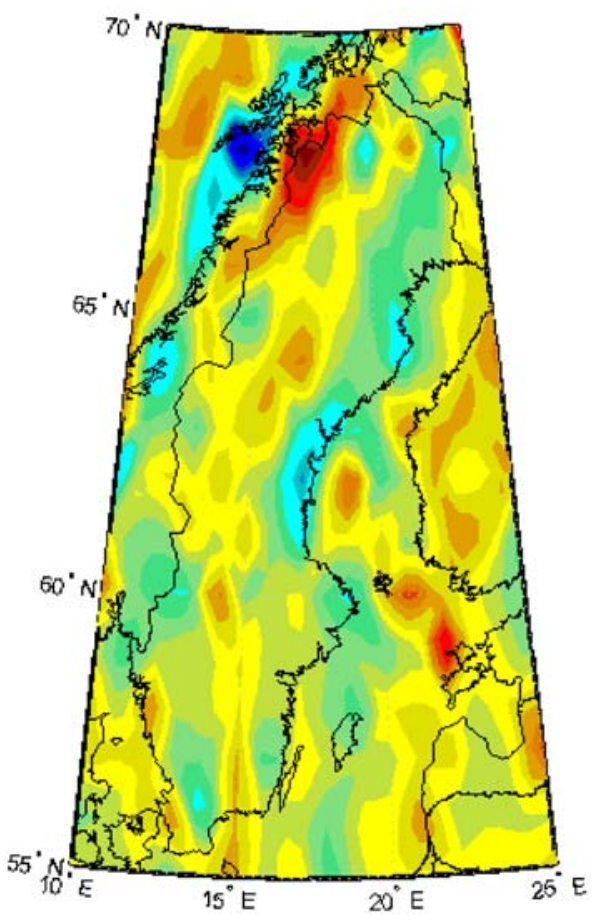
Tyz,Unit: Eotvos e
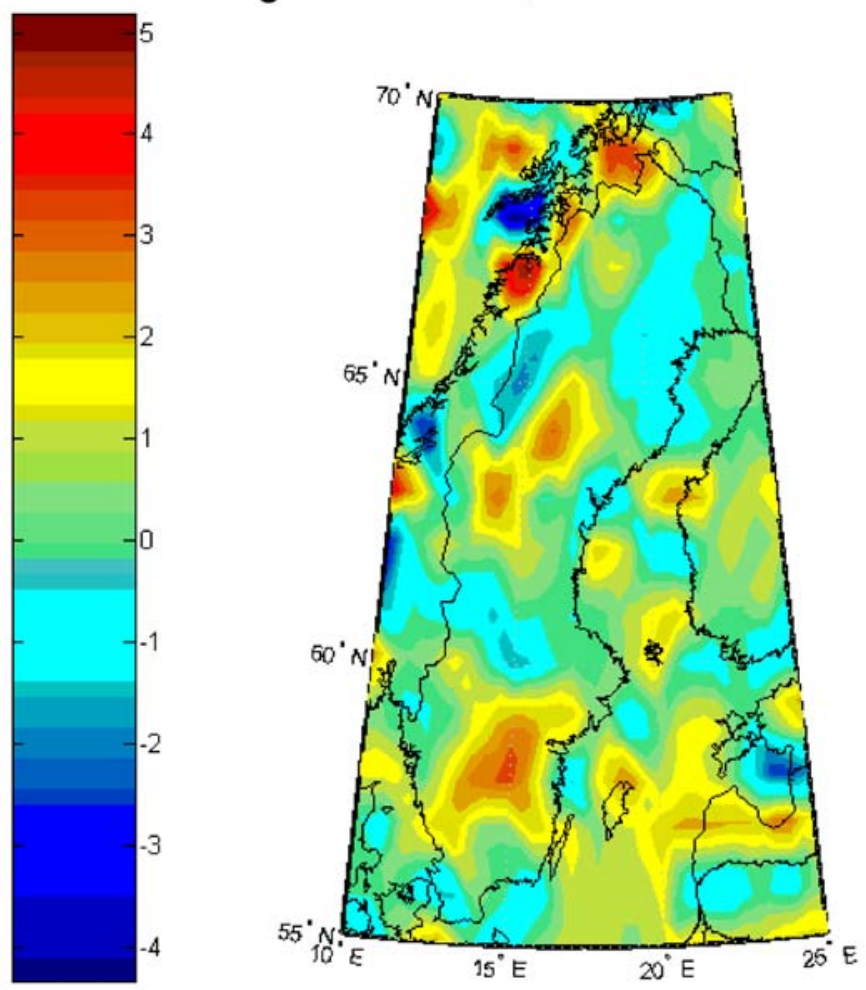

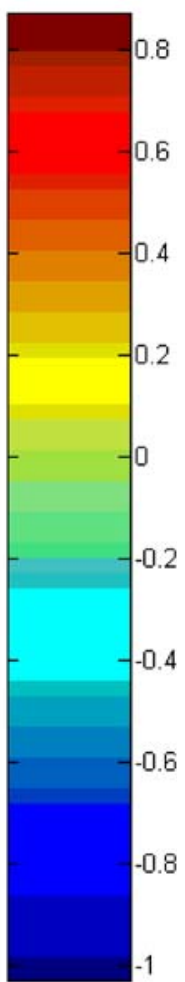

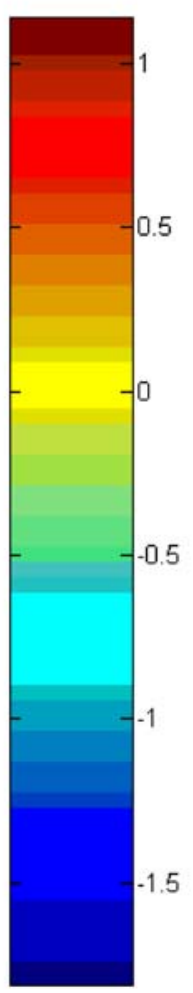

Fig. 3 (continued) 
maximum degree plus two. The out put of this function is all needed coefficients for computing the gravity gradient coefficients.

\section{- Ngrav function}

This function computes the normal gravity and its derivative with respect to latitude on the reference ellipsoid. This function is applied for generating geoid and deflection of verticals as it is seen in Eqs. 3, 4, and 5. The input of this function is latitude. The computation depends on the Somigliana's formula (Moritz 2000), which is the most accurate one among the others (e.g., first and second Chebyshev approximations), and depends on the GRS80 ellipsoid, which is the more up-to-date and internationally adopted reference ellipsoid (Featherstone and Dentith 1997; Vanicek and Krakiwsky 1986).

\section{- Delta function}

This function is to compute the Kronecker's delta.

When spherical harmonic expansions are used for gravity field modelling, the numerical efficiency of the algorithm, the stability of the recurrence relations that are used to compute the associated Legendre functions and the under-flow of early values in the recurrence relations must be taken into account (Wittwer et al. 2008). The numerical performance of EGMLab program is tested and verified with the performance of publicly independent solutions of ICGEM and American National Geospatial-Intelligence Agency (NGA) EGM96 software (http://earth-info.nga. $\mathrm{mil} / \mathrm{GandG} / \mathrm{wgs} 84 /$ gravitymod/egm96/egm96.html). The results of investigation show that the performance of the EGMLab up to maximum degree and order 360 is better than EGM96 official software. Then we want to investigate the numerical stability of EGMLab program. The correctness of geoidal height parameter was checked by synthesizing gravity potential values up to degree 360 on a global grid, using EGM96 potential model. These gravity potential values were then used to estimate the original potential coefficients using spherical harmonic analysis with the program. The resulting error degree variances were in the range of eight to 12, the magnitude of numerical accuracy. Furthermore, the computation of the geoidal height parameter was compared for various degrees and latitudes with the EGM96 values. As expected, the geoidal height errors are very small, with amplitudes $<1 \mathrm{~mm}$. Therefore, we safely assume both programs to work correctly up to degree 360 or even 720 . All tests were performed on an Intel Pentium4 clocked at $3 \mathrm{GHz}$, using MATLAB 7.2 under Windows VISTA. All programs were run single-threaded in 32 bit-modes.

It is important to mention here that the NGA and ICGEM software/website are provided just for the special GGMs (e.g. EGM96) or have limitations in determination of the point-wise data and specially gradient components parameters. Comparing the mentioned publicly web-based/ commercial software, the EGMlab is a more user friendly software which can be used for determination of the most of gravity or gradient components parameters from any GGM models. The EGMLab is freeware/non-commercial "As-is" software and can be downloaded from the Department of Geodesy and Gematics of Zanjan University website from the following Internet link: http://www. znu.ac.ir/members/kiamehr_ramin/kiamehr/egmlab.zip). Although EGMlab and EGM96 software are free, they run under commercial software (e.g. FORTRAN and MATLAB) which are usually available in most academic and research institutes.

Our investigations on the pre-release version of the EGM2008 show that EGMlab can be used successfully up to degrees and orders 720 for estimation of any gravity components. However, the associated Legendre functions are generated recursively and the corresponding recursive relation are unstable at very high degrees. In such cases, Horner and Clenshaw algorithms are preferable, although we have not used such algorithms in our software. This kind of computation still is under testing and development, and from the computational efficiency point of view it is too slow and time consuming. However, our investigation on the JAVA based version of the EGM2008 software on ICGEM website clearly indicates that estimation of any gravity components parameter (up to 2,160), requires the user to wait at least an hour in order to get any results.

\section{A sample numerical results over Sweden}

In order to show the potential of the EGMlab software, we present the gravity and gradient components of the gravity field based on the EIGEN-GL04C GRACE model (up to degrees and orders 360) in Sweden. Figure 2 and Table 1 present results of basic gravity components parameters which are estimated using the EGMlab1. Also, in Fig. 3 and Table 2 we present the results of estimation for

Table 2 Statistical analysis of gradient components values in Sweden based on the EIGEN-GL04C model (unit: Eotvos)

\begin{tabular}{lrrrr}
\hline Gradient components & \multicolumn{1}{l}{ Min } & \multicolumn{1}{l}{ Max } & \multicolumn{1}{l}{ Mean } & \multicolumn{1}{l}{ Std } \\
\hline$T_{x x}$ & -20.745 & 23.913 & 0.350 & 5.268 \\
$T_{y y}$ & -1.323 & 1.447 & 0.031 & 0.337 \\
$T_{z z}$ & -25.359 & 22.068 & -0.382 & 5.563 \\
$T_{x y}$ & -5.623 & 7.479 & 0.000 & 1.734 \\
$T_{x z}$ & -1.240 & 1.172 & -0.025 & 0.328 \\
$T_{y z}$ & -2.080 & 1.381 & -0.038 & 0.409 \\
\hline
\end{tabular}


the gradient components parameters using the EGMlab3. Based on the purpose of user, it is possible to use the output text file for different purposes e.g., geoid modelling, comparisons between GGMs to finding the model in the study area, evaluation of the gravity anomalies and filling the possible gaps. Also, the deflection of vertical deflections and gradient components can be used in geophysical interpretations.

Figure 2a shows the long wavelength structure of the geoid in Sweden generated by the software. Figure $2 b$ is very similar to Fig. $3 \mathrm{C}$ as they illustrate the maps of gravity anomaly and vertical-vertical components of the gravity gradients tensor. As we know, these two quantities are utilized for exploration geophysics to determine depth of the anomalous features. Figure $3 \mathrm{~A}$ and $\mathrm{B}$ show the maximum curvature of the equipotential surface along north and west, respectively. They can detect the slide of the anomalous features in these two directions. Figure 3E and $\mathrm{F}$ can be interpreted as Fig. $2 \mathrm{c}$ and $\mathrm{d}$ and they present the boundaries of the features too. Figure $3 \mathrm{D}$ has more complicated interpretation than the other quantities showing the corners of the features. Comparing the gravity gradients maps and statistics, one can conclude that $T_{x y}$ has the smallest magnitude among others as it does not include zero- and first-degree harmonic coefficients in its expression; after that $T_{x z}$ and $T_{y z}$ are smallest because of excluding the zero-degree harmonic. The results are promising as the gravity gradients are very sensitive to the local interior features. It is obvious that the sensitivity will be reduced by elevation, e.g. at airborne or even satellite level, and the long wavelength structure of the regional features can be investigated more.

\section{Evaluation using GPS/levelling data}

In this section the model is evaluated using GPS/levelling observations, and a comparison is made with the GL04C GGM. To evaluate the result of EGMlab, 195 highquality GPS/ levelling height anomalies in the reference systems SWEREF 99 and RH 2000 are available; the locations are illustrated on the maps of Fig. 4. The orthometric heights in RH 2000 have been determined by utilising high quality levelling connections. The coordinates of the SWEPOS stations are very well determined. GPS heights have been derived using at least $48 \mathrm{~h}$ of observations. The GPS/levelling residuals after a oneparameter transformation (fit) of the GL04C model is plotted in Fig. 4. The statistics after both a one- and a fourparameter transformation are given in Table 3. As can be seen in Fig. 4, the agreement is good and homogeneous for the whole of Sweden. The results clearly show that the differences from not using exactly the same data are very small based on the GL04C GGM.

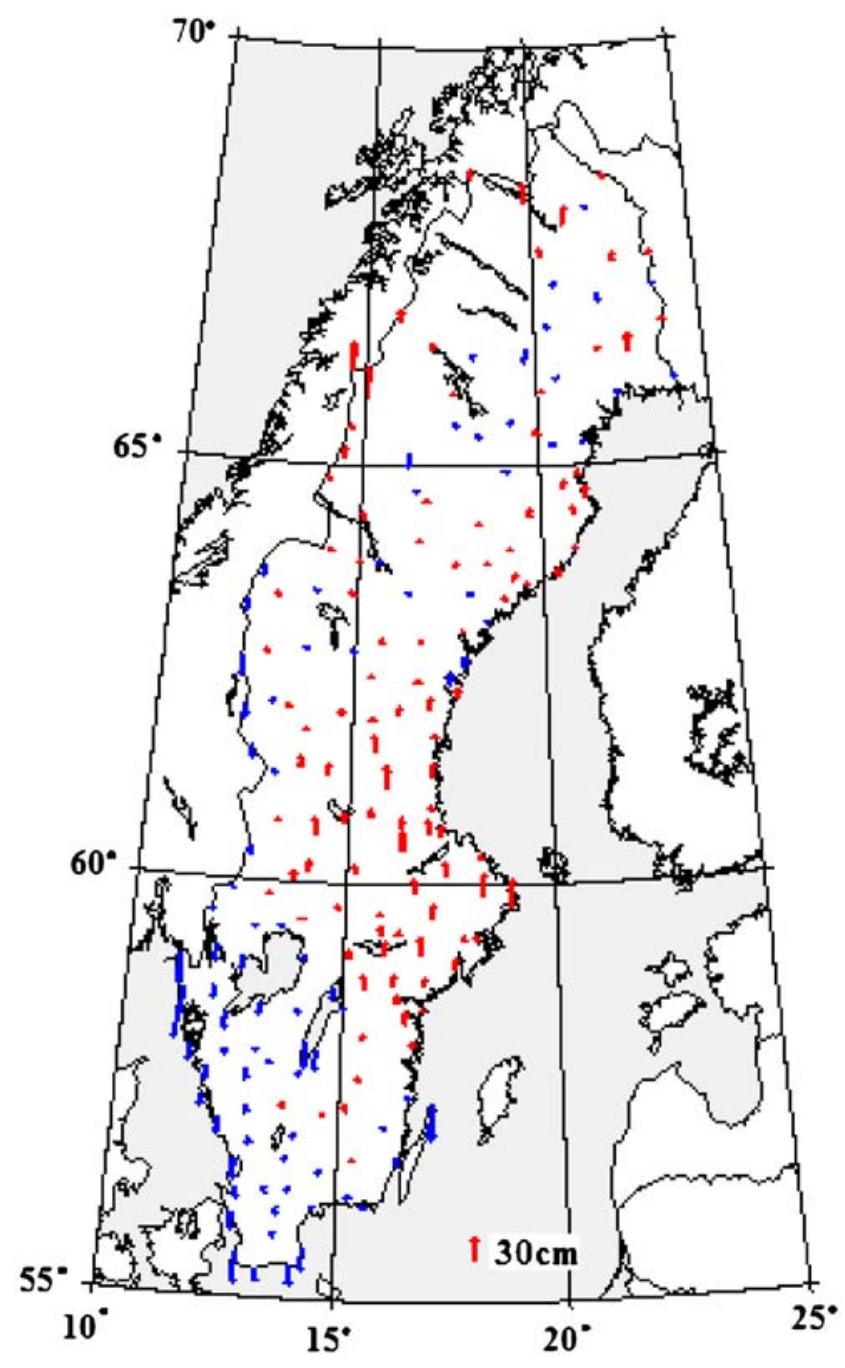

Fig. 4 GPS/levelling residuals for the GL04C model

\section{Conclusions}

In this article, determining the gravity and gradient components of the gravity field using the GGMs is reviewed. Then article is followed by a numerical case study based on the EIGEN-GL04C GRACE GGM. We presented a software program for spherical harmonic analysis, which can essentially be used for degrees and order up to 720 and all latitudes in the interval $\left(0^{\circ}, \pm 90^{\circ}\right)$. The provided software and methods can be used for

Table 3 Comparison of the EGMlab results with GNSS/levelling

\begin{tabular}{lccccc}
\hline GGM & No. of parameters & Min & Max & Mean & SE \\
\hline GL04C & 1 & -0.493 & 0.340 & 0 & 0.127 \\
& 4 & -0.358 & 0.367 & 0 & 0.110
\end{tabular}

Statistics for the 195 GPS/levelling residuals after a one- and a fourparameter fit. Unit: $\mathrm{m}$ 
evaluation of the recent GRACE based GGMs for the gravimetric geoid modelling, evaluation of the gravity anomaly data and for a scientific analysis of the gradient for possible simulation for GOCE satellite data. The EGMLab can be easily used with any new GGMs just by changing the spherical harmonics coefficients files.

Acknowledgments The valuable suggestions and comments from Prof. Hassan A. Babaie and three anonymous reviewers are greatly appreciated. The authors are grateful to Mr. Mohammad Reza Taghiloo for the English review of this paper.

\section{References}

Eshagh M, Alamdari MN, Farnin A (2006) Investigation of long wavelength spheroids of EIGEN geopotential models in Iran. Proceedings of the FIG conference, Sofia, Bulgaria

Featherstone W (1999) The use and abuse of vertical deflections. Proceedings of the Sixth South East Asian Surveyors' Congress, Fremantle, Western Australia

Featherstone WE, Dentith MC (1997) A geodetic approach to gravity data reduction for geophysics. Comput Geosci 23(10):1063-1070

Förste C, Schmidt R, Stubenvoll R, Flechtner F, Meyer U, Stubenvoll R, Rothacher M, König R, Neumayer H, Biancale R, Lemoine J-M, Bruinsma S, Loyer S, Barthelmes, Neumayer F (2006) A mean global gravity field model from the combination of satellite mission and altimetry/ gravimetry surface data-EIGEN-GL04C. Geophysical Research Abstracts 8:03462

Förste C, Schmidt R, Stubenvoll R, Flechtner F, Meyer U, König R, Neumayer H, Biancale R, Lemoine J-M, Bruinsma S, Loyer S, Barthelmes F, Esselborn S (2007) The GeoForschungsZentrum Potsdam/Groupe de Recherche de Geodesie Spatiale satellite-only and combined gravity field models: EIGEN-GL04S1 and EIGENGL04C. J Geod 82:331-346. doi:10.1007/s00190-007-0183-8

Heiskanen WA, Moritz H (1967) Physical geodesy. Freeman, San Francisco

Kiamehr R (2006) The impact of the lateral density variation model in determination of the precise gravimetric geoid in mountainous areas: a case study of Iran. Geophysical Research Abstracts 8:01377
Kiamehr R, Sjöberg LE (2005) The qualities of Iranian gravimetric geoid models versus recent gravity field missions. Stud Geophys Geod 49:289-304

Kiamehr R, Eshagh M, Sjöberg LE (2008) Interpretation of general geophysical patterns in Iran based on GRACE gradient component analysis. Acta Geophysica 56(2):440-454

Kirby JF, Featherstone WE, Kearsley AHW (1998) Tests of the DMA/GSFC geopotential models over Australia. Geophys J Int $7: 2-13$

Lemoine FG, Smith D, Smith R, Kunz, Pavlis E, Pavlis N, Klosko S, Chinn D, Torrence M, Williamson R, Cox C, Rachlin K, Wang Y, Kenyon S, Salman R, Trimmer R, Rapp R, Nerem S (1996) The development of the NASA GSFC and DMA joint geopotential model. Proceedings of the Symposium on Gravity, Geoid and Marine Geodesy, September 30-October 5, The University of Tokyo, Tokyo

Moritz H (2000) Geodetic reference system (1980). J Geod (74/ 1):128-162

Petrovskaya MS, Vershkov AN (2006) Non-singular expressions for the gravity gradients in the local north-oriented and orbital references frames. J Geod 80:117-127

Pavlis NK, Holmes SA, Kenyon SC, Factor JK (2008) An Earth gravitational model to degree 2160: EGM2008. Presented at the 2008 General Assembly of the European Geosciences Union, Vienna, Austria, April 13-18

Prange L, Jäggi A, Beutler G, Mervart L, Dach R (2007) Gravity field determination at the AIUB - the Celestial Mechanics Approach. Poster presented at the IUGG XXIV General Assembly, July 213, Perugia/Italy

Reed GB (1973) Application of kinematical geodesy for determining the shorts wave length components of the gravity field by satellite gradiometry. Ohio State University, Department of Geodesy, Science, Report No. 201, Columbus, Ohio

Tapley B, Ries J, Bettadpur S, Chambers D, Cheng M, Condi F, Gunter B, Kang Z, Nagel P, Pastor R, Pekker T, Poole S, Wang F (2005) GGM02 - an improved Earth gravity field model from GRACE. J Geod 79:467-478 doi:10.1007/s00190-005-0480-z

Vanicek P, Krakiwsky EJ (1986) Geodesy: the concepts. NorthHolland, Amsterdam

Wenzel HG (1998) Ultra-high degree geopotential models GPM98A and GPM98B to degree 1800. Report 98:4, Finnish Geodetic Institute, Masala, pp 71-80

Wittwer T, Klees R, Seitz K, Heck B (2008) Ultra-high degree spherical harmonic analysis and synthesis using extended-range arithmetic. J Geod 82:223-229 